\title{
Long noncoding RNA OSER1-AS1 promotes the malignant properties of non-small cell lung cancer by sponging microRNA-433-3p and thereby increasing Smad2 expression
}

\author{
XINMEI LIU ${ }^{1}$, SHASHA HUANG ${ }^{2}$, YUN GUAN $^{1}$ and QING ZHANG ${ }^{1}$ \\ Departments of ${ }^{1}$ Respiratory Disease and ${ }^{2}$ General Surgery, Weifang People's Hospital, Weifang, Shandong 261041, P.R. China
}

Received February 28, 2020; Accepted May 20, 2020

DOI: $10.3892 /$ or.2020.7645

\begin{abstract}
OSER1 antisense RNA 1 (OSER1-AS1), a long noncoding RNA, has been well studied in the context of hepatocellular carcinoma. However, its expression status, specific functions, and tumorigenic mechanism in non-small cell lung cancer (NSCLC) remain uninvestigated. Hence, this study aimed to assess OSER1-AS1 expression, test the malignancy-related biological functions of OSER1-AS1, and illustrate how they affect NSCLC progression. OSER1-AS1 expression in NSCLC was measured by reverse transcription-quantitative polymerase chain reaction. Cell Counting Kit- 8 assay, flow cytometry, cell migration and invasion assay, and tumor xenograft assay were performed to analyze the effects of OSER1-AS1 on the malignant phenotypes of NSCLC cells. Bioinformatics prediction with luciferase reporter and RNA immunoprecipitation assays were performed to determine the interaction between OSER1-AS1 and microRNA-433-3p (miR-433-3p). OSER1-AS1 was strongly expressed in NSCLC tissues and cell lines. Enhanced OSER1-AS1 expression was significantly correlated with tumor size, TNM stage, and lymph node metastasis in patients with NSCLC. Patients with NSCLC exhibiting high OSER1-AS1 expression had shorter overall survival than those exhibiting low OSER1-AS1 expression. Functionally, a reduction in OSER1-AS1 expression led to significant decreases in NSCLC cell proliferation, migration, and invasion as well as an increase in cell apoptosis in vivo. OSER1-AS1 knockdown suppressed the tumorigenic ability of NSCLC cells in vivo. Mechanistically, OSER1-AS1 acts as a competing endogenous RNA (ceRNA) in NSCLC cells by sponging miR-433-3p and thereby increasing the expression of mothers against decapentaplegic homolog 2 (Smad2). Finally, restoration experiments revealed that the suppression of miR-433-3p and restoration of Smad2 both counteracted
\end{abstract}

Correspondence to: Professor Qing Zhang, Department of Respiratory Disease, Weifang People's Hospital, 151 Guangwen Road, Weifang, Shandong 261041, P.R. China

E-mail: zhangqin_shandong@163.com

Key words: OSER1 antisense RNA 1, non-small cell lung cancer, microRNA-433-3p, mothers against decapentaplegic homolog 2 the suppressive effects of OSER1-AS1 depletion in NSCLC cells. Our findings illustrate the biological importance of the OSER1-AS1/miR-433-3p/Smad2 pathway in NSCLC progression and offer a novel perspective regarding the identification of effective therapeutic and diagnostic targets.

\section{Introduction}

Lung cancer is the most common malignancy and the leading cause of cancer-related deaths worldwide (1). Globally, approximately 228,150 newly diagnosed non-small cell lung cancer (NSCLC) cases and 147,510 deaths due to NSCLC are reported annually (2). NSCLC, which is associated with high morbidity and mortality rates, accounts for $80-85 \%$ of all lung cancer cases (3). NSCLC is classified into three categories, one with actionable oncogene mutations including epidermal growth factor receptor ( $E G F R$ ), Anaplastic lymphoma kinase $(A L K)$ and ROS proto-oncogene 1, receptor tyrosine kinase (ROS1), one which responds to immune checkpoint inhibitors, and others (4,5). Many patients with NSCLC present with local or distant metastasis at the time of initial diagnosis, and this is attributed to atypical clinical manifestations and lack of specific diagnostic techniques in the early stage of the disease (6). At present, surgical resection and chemoradiotherapy are applied as first-line therapeutics for NSCLC (7). Despite considerable developments in the diagnosis and treatment of NSCLC in the last decade, including surgical excision, radiotherapy, chemotherapy and targeted therapy, no noticeable improvements have been made in patient outcomes, with the 5-year survival rate being only $15 \%$ (8). Tremendous efforts have been made to elucidate the mechanisms associated with NSCLC pathogenesis $(9,10)$. Hence, the identification of novel and promising therapeutic targets is urgent and helpful for the promotion of NSCLC treatment.

Recently, long noncoding RNAs (IncRNAs) have received considerable attention in the field of cancer research (11). These IncRNAs are defined as a family of transcripts with a characteristic length exceeding 200 nucleotides and the absence of an open reading frame (12). Although these lncRNAs do not encode proteins, an increasing number of studies have illustrated their crucial roles as regulators of nearly all cellular processes as well as of carcinogenesis and cancer progression $(13,14)$. In particular, reports have described the dysregulation of many lncRNAs in NSCLC. For 
example, LINC00514 (15), PVT1 (16), and MIR503HG expression are upregulated in NSCLC, whereas LINC00261 (17), TOB1-AS1 (18), and NBR2 (19) are expressed at low levels. lncRNAs may act as tumor suppressors or promoters of the oncogenicity of NSCLC, and they have been identified in the regulation of multiple aggressive phenotypes $(20,21)$.

MicroRNAs (miRNAs) are a group of endogenous, highly conserved, and noncoding RNA molecules comprising 17-24 nucleotides. miRNAs have been implicated in the negative modulation of gene expression via the inhibition of messenger RNA (mRNA) translation and/or induction of mRNA degradation through direct binding to the complementary sites in 3'-untranslated regions of their target genes (22). lncRNAs may function as competing endogenous RNAs (ceRNAs) to control gene expression by competitively binding to miRNA response elements (23). Therefore, lncRNAs and miRNAs may exert increasingly crucial functions in terms of the early diagnosis and molecular-targeted therapy of NSCLC.

Although OSER1 antisense RNA 1 (OSER1-AS1) has been well studied in hepatocellular carcinoma (24), its expression status, specific functions, and tumorigenic mechanism in NSCLC remain unknown. Accordingly, we aimed to assess OSER1-AS1 expression in NSCLC, test the biological functions of OSER1-AS1 associated with NSCLC, and illustrate how they affect NSCLC progression.

\section{Materials and methods}

Human tissue samples. This study was approved by the Ethics Committee of Weifang People's Hospital and was performed in accordance with the principles of the Declaration of Helsinki. In total, 53 patients with NSCLC (22 male and 31 female patients; age range, 59-73 years) admitted to Weifang People's Hospital participated in this study between March 2014 to January 2015 and provided written informed consent. None of the enrolled patients had previously received preoperative radiotherapy, chemotherapy, targeted therapy, or any other anticancer treatments. The standard care of patients with NSCLC is chemoradiotherapy for stage III and chemotherapy for stage IV. Previous studies have reported that cytoreductive surgery can improve the quality of life of patients with advanced or distant metastasis of lung cancer $(25,26)$; hence, the 29 NSCLC patients diagnosed with stage III-IV admitted in our study received cytoreductive surgery. NSCLC tissues and adjacent normal tissues were obtained after surgical resection and immediately frozen and conserved in liquid nitrogen.

Cell culture. The human nontumorigenic bronchial epithelial cell line BEAS-2B was purchased from the Shanghai Academy of Life Science (Shanghai, China) and cultured in the BEGM ${ }^{\mathrm{TM}}$ Bronchial Epithelial Cell Growth Medium (Lonza/Clonetics Corporation) supplemented with $0.5 \mathrm{ng} / \mathrm{ml}$ epidermal growth factor, $500 \mathrm{ng} / \mathrm{ml}$ hydrocortisone, $0.035 \mathrm{ng} / \mathrm{ml}$ bovine pituitary extract, $500 \mathrm{mM}$ ethanolamine, $500 \mathrm{nM}$ ethanolamine phosphate, $0.01 \mathrm{mg} / \mathrm{ml}$ adrenaline, and $0.1 \mathrm{~g} / \mathrm{ml}$ retionic acid.

Five human NSCLC cell lines, namely, H522 (human lung adenocarcinoma cell carcinoma), H460 (human lung large cell carcinoma), H1703 (human lung squamous cell carcinoma), A549 (NSCLC), and SK-MES-1 (human lung squamous cell carcinoma), were also obtained from the Shanghai Academy of Life Science. The cell lines H522, H460, H1703, and A549 were maintained in RPMI-1640 media (Gibco/Thermo Fisher Scientific, Inc.) containing 10\% fetal bovine serum (FBS; Gibco/Thermo Fisher Scientific, Inc.) and $1 \%$ penicillin/streptomycin (Gibco/Thermo Fisher Scientific, Inc.). SK-MES-1 cells were cultured in Minimal Essential Medium (Gibco/Thermo Fisher Scientific, Inc.) supplemented with $10 \% \mathrm{FBS}$ and $1 \%$ penicillin/streptomycin. All cells were cultured at $37^{\circ} \mathrm{C}$ in an incubator supplied with $5 \% \mathrm{CO}_{2}$.

Transfection. The small interfering RNAs (siRNAs) used to silence OSER1-AS1 (si-OSER1-AS1\#1, si-OSER1-AS1\#2 and si-OSER1-AS1\#3) and negative control (NC) siRNA (si-NC) were synthesized by Genepharma. miR-433-3p mimic, NC miRNA mimic (miR-NC), miR-433-3p inhibitor (anti-miR-433-3p), and NC miRNA inhibitor (anti-miR-NC) were obtained from RiboBio. The pcDNA3.1 empty vector and pcDNA3.1-Smad2 overexpression vector (pc-Smad2) were designed and produced by GeneChem. Transient transfection was conducted using the Lipofectamine $2000^{\mathrm{TM}}$ reagent (Invitrogen/Thermo Fisher Scientific, Inc.).

Reverse transcription-quantitative polymerase chain reaction $(R T-q P C R)$. Total RNA was extracted from tissue samples or cells using the RNeasy Mini Kit (Qiagen). The purity and concentration of total RNA were analyzed using the DeNovix DS-11 Spectrophotometer (DeNovix, Inc.). Reverse transcription of OSER1-AS1 and Smad2 mRNA was performed using the PrimeScript RT Reagent Kit (Takara). The synthesized cDNA was subjected to quantitative polymerase chain reaction (qPCR) using the SYBR Premix Ex Taq (Takara). To quantify miR-433-3p expression, total RNA was reverse transcribed into cDNA using the miRcute miRNA First-Strand cDNA Synthesis Kit (Tiangen Biotech). Next, qPCR was conducted using the miRcute miRNA qPCR Detection Kit with SYBR Green (Tiangen Biotech). Glyceraldehyde-3-phosphate dehydrogenase $(G A P D H)$ was used as the internal control for the normalization of OSER1-AS1 and Smad2 mRNA expression, whereas U6 small nuclear RNA was used for the normalization of miR-433-3p expression. Relative gene expression levels were analyzed using the $2^{-\Delta \Delta C q}$ method (27).

Nuclear and cytoplasmic RNA fractionation. The PARIS Kit (Invitrogen/Thermo Fisher Scientific, Inc.) was used for nuclear and cytoplasmic RNA fractionation. The contents of OSER1-AS1 in nuclear and cytoplasmic fractions were quantified using RT-qPCR. GAPDH and U6 were used as internal controls to assess fractioning efficiency.

Cell Counting Kit-8 (CCK-8) assay. Transfected cells that had been incubated at $37^{\circ} \mathrm{C}$ in an incubator supplied with $5 \% \mathrm{CO}_{2}$ for $24 \mathrm{~h}$ were harvested, resuspended in a culture medium, and seeded into $96-$-well plates at a density of $2 \times 10^{3}$ cells/well. Four post-inoculation time points were set: $0,24,48$, and $72 \mathrm{~h}$. At each time point, cell proliferation was assessed by incubating cells with $10 \mu \mathrm{l}$ of CCK-8 solution (Sigma-Aldrich; Merck $\mathrm{KGaA}$ ) at $37^{\circ} \mathrm{C}$ for $2 \mathrm{~h}$, after which the optical density was measured at $450-\mathrm{nm}$ wavelength using a Tecan microplate reader (Tecan Group, Ltd.). 

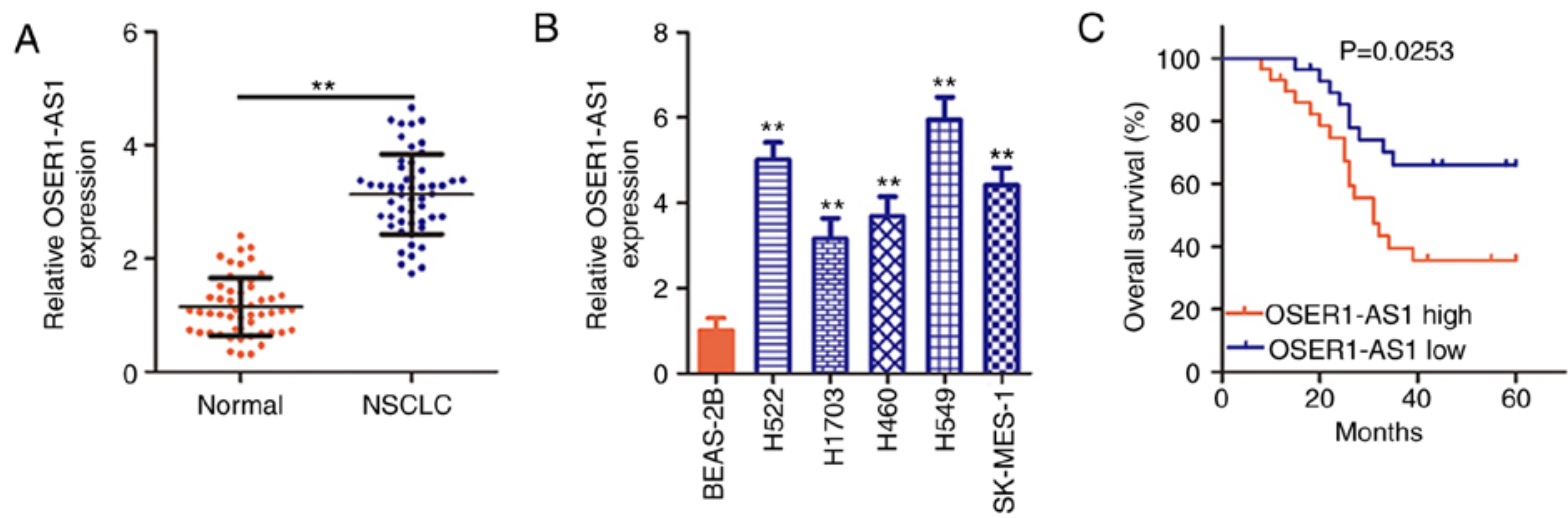

Figure 1. Upregulation of the expression of long noncoding RNA OSER1-AS1 in NSCLC is associated with poor prognosis. (A) Evaluation of OSER1-AS1 expression in 53 pairs of NSCLC tissues and adjacent normal tissues by RT-qPCR. ${ }^{* *} \mathrm{P}<0.01$, compared with the adjacent normal tissues. (B) Clarification of the OSER1-AS1 expression status in five human NSCLC cell lines (H522, H460, H1703, A549, and SK-MES-1) using RT-qPCR. The human nontumorigenic bronchial epithelial cell line BEAS-2B served as the control. ${ }^{* *} \mathrm{P}<0.01$, compared with the BEAS-2B cells. (C) Comparison of the overall survival of patients with NSCLC in the high- and low-OSER1-AS1 expression groups ( $\mathrm{P}=0.0253)$. OSER1-AS1, RNA OSER1 antisense RNA 1; NSCLC, non-small cell lung cancer; RT-qPCR, reverse transcription-quantitative polymerase chain reaction.

Flow cytometry. An Annexin V-fluorescein isothiocyanate (FITC) apoptosis detection kit (BioLegend, Inc.) was used to evaluate cell apoptosis. After $48 \mathrm{~h}$ of culture, transfected cells were digested with an ethylenediaminetetraacetic acid-free trypsin reagent and centrifuged at $12,000 \mathrm{x} \mathrm{g}$, followed by two washes with ice-cold phosphate-buffered solution and resuspension in $100 \mu 1$ of $1 \mathrm{X}$ binding buffer. Next, the cells were stained with $5 \mu \mathrm{l}$ Annexin V-FITC and $5 \mu \mathrm{l}$ propidium iodide (PI) for $15 \mathrm{~min}$ at room temperature in the dark. Then, the cells were analyzed using a flow cytometer (FACScan; BD Biosciences) equipped with the CellQuest software (version 2.9; $\mathrm{BD}$ Biosciences) to determine the frequency of cell apoptosis.

Cell migration and invasion assays. Transfected cells were trypsinized, washed, centrifuged, and collected. The cells were mixed with FBS-free culture medium to yield a cell suspension at a density of $1 \times 10^{5}$ cells $/ \mathrm{ml}$. Regarding cell migration assay, $100 \mu \mathrm{l}$ of the suspension was added to each upper chamber of wells equipped with $8-\mu \mathrm{m}$ porous membranes (BD Biosciences), whereas $600 \mu \mathrm{l}$ of complete culture medium was added to each lower chamber to induce migration. After $24 \mathrm{~h}$ of incubation at $37^{\circ} \mathrm{C}$ in an incubator supplied with $5 \% \mathrm{CO}_{2}$, the cells that had not migrated to the lower chamber were removed with a cotton swab, whereas the migrated cells were fixed for 20 min with $4 \%$ (v/v) paraformaldehyde and stained for 20 min with $0.1 \%$ crystal violet. The number of migrated cells was counted in five randomly selected fields under an inverted microscope (magnification, x100; Olympus Corp.). Regarding the cell invasion assay, chambers were precoated with Matrigel (BD Biosciences). All subsequent steps were performed as described for the cell migration assay.

Tumor xenograft assay. The short hairpin RNAs (shRNAs) targeting OSER1-AS1 (sh-OSER1-AS1) and NC shRNA (sh-NC) were inserted into the pLKO.1 vector. After lentivirus production, $\mathrm{H} 522$ cells were injected with lentivirus expressing sh-OSER1-AS1 or sh-NC. Puromycin selection was then performed to obtain stably transfected cells.

The protocol for animal experiments was ratified by the Animal Ethics Committee of the Weifang People's
Hospital. Regarding tumor xenograft assay, a total of 6 4- to 6-week-old male BALB/c nude mice $(20 \mathrm{~g}$; Vital River Laboratory Animal Technology, Beijing, China) were randomly divided into two groups: sh-OSER1-AS1 $(n=3)$ and sh-NC $(n=3)$. Mice in the sh-OSER1-AS1 and sh-NC groups were subcutaneously inoculated with $5 \times 10^{6} \mathrm{H} 522$ cells stably expressing sh-OSER1-AS1 and sh-NC, respectively. All mice were housed under specific pathogen-free conditions at $25^{\circ} \mathrm{C}$ with $50 \%$ humidity, with a $10 / 14-\mathrm{h}$ light/dark cycle and ad libitum food/water access. The width and length of each tumor xenograft were measured weekly beginning at postinjection 7 days, and tumor volume $(\mathrm{V})$ was calculated using the following formula: $\mathrm{V}=\left(\right.$ length $\mathrm{x}$ width $\left.{ }^{2}\right) / 2$. At postinjection 5 weeks, mice were euthanized via cervical dislocation. All tumor xenografts were dissected, photographed, and weighed using an analytical balance. Subsequently, total RNA and protein were isolated from the tumor xenografts for RT-qPCR and western blotting, respectively, according to the aforementioned protocols.

IncRNA-miRNA interactions. Putative miRNAs that directly interact with OSER1-AS1 were predicted using the public database StarBase 3.0 online tool (http://starbase.sysu.edu.cn/) (28).

RNA immunoprecipitation (RIP) assay. RIP was performed using the Magna RIP RNA-binding protein immunoprecipitation kit (Millipore) in accordance with the product instructions. Briefly, cells were incubated with RIP lysis buffer supplemented with a protease inhibitor cocktail and an RNase inhibitor. Then, the cell extract was incubated with magnetic beads conjugated with anti-Argonaute (anti-Ago2) or anti-IgG antibodies (dilution 1:5,000; both from cat. no. 03-110; Millipore). IgG was used as NC. After overnight incubation at $4^{\circ} \mathrm{C}$, coprecipitated RNA was extracted and subjected to RT-qPCR to determine the enrichment of OSER1-AS1 and miR-433-3p.

Luciferase reporter assay. The OSER1-AS1 fragments carrying predicted wild-type miR-433-3p-binding sequences or mutant OSER1-AS1 fragments were amplified and 
Table I. Association between the clinicopathological factors of the NSCLC patients and OSER1-AS1 expression.

\begin{tabular}{|c|c|c|c|}
\hline \multirow[b]{2}{*}{$\begin{array}{l}\text { Clinicopathological } \\
\text { factors }\end{array}$} & \multicolumn{2}{|c|}{$\begin{array}{c}\text { OSER1-AS1 } \\
\text { expression }(\mathrm{n}=53)\end{array}$} & \multirow[b]{2}{*}{ P-value } \\
\hline & $\begin{array}{l}\text { High } \\
(n=27)\end{array}$ & $\begin{array}{l}\text { Low } \\
(\mathrm{n}=26)\end{array}$ & \\
\hline Sex & & & 0.782 \\
\hline Male & 12 & 10 & \\
\hline Female & 15 & 16 & \\
\hline Age (years) & & & 0.264 \\
\hline$<65$ & 8 & 12 & \\
\hline$\geq 65$ & 19 & 14 & \\
\hline Classification & & & 0.553 \\
\hline Adenocarcinoma & 11 & 9 & \\
\hline Squamous & 12 & 15 & \\
\hline Large cell & 4 & 2 & \\
\hline Differentiation & & & 0.782 \\
\hline Well + Moderate & 10 & 11 & \\
\hline Poor & 17 & 15 & \\
\hline Tumor size $(\mathrm{cm})$ & & & 0.024 \\
\hline$<3$ & 12 & 20 & \\
\hline$\geq 3$ & 15 & 6 & \\
\hline TNM stage & & & 0.028 \\
\hline I-II & 8 & 16 & \\
\hline III-IV & 19 & 10 & \\
\hline Lymph node metastasis & & & 0.045 \\
\hline Negative & 13 & 20 & \\
\hline Positive & 14 & 6 & \\
\hline
\end{tabular}

NSCLC, non-small cell lung cancer; OSER1-AS1, OSER1 antisense RNA 1.P-values in bold print indicate significant differences.

inserted into the psiCHECK-2 plasmid (Promega Corp.). The resulting luciferase reporter plasmids were labeled as OSER1-AS1-wt and OSER1-AS1-mut, respectively. Cells were seeded in 24-well plates at a density of $1.5 \times 10^{5}$ cells per well and transfected with OSER1-AS1-wt or OSER1-AS1-mut in combination with miR-433-3p mimic or miR-NC using the Lipofectamine $2000^{\mathrm{TM}}$ reagent. After $48 \mathrm{~h}$ of incubation at $37^{\circ} \mathrm{C}$ in an incubator supplied with $5 \% \mathrm{CO}_{2}$, the transfected cells were rinsed with phosphate-buffered saline. Luciferase activity was measured using the Dual-Luciferase Reporter Assay System (Promega Corp.). Renilla luciferase activity was used to normalize the level of firefly luciferase activity.

Western blotting. Concentrations of proteins in cells lysed in RIPA buffer (Beyotime Institute of Biotechnology) were determined using a BCA protein kit (Beyotime Institute of Biotechnology). Equal amounts of protein $(30 \mu \mathrm{g})$ were loaded into each lane of a $10 \%$ sodium dodecyl sulfate polyacrylamide gel, electrophoresed, and transferred onto polyvinylidene difluoride membranes. The membranes were blocked for $2 \mathrm{~h}$ in $5 \%$ fat-free milk at room temperature and incubated with primary antibodies against Smad2 (cat. no. ab40855; 1:1,000 dilution; Abcam) or GAPDH (cat. no. ab181602; 1:1,000 dilution; Abcam) overnight at $4^{\circ} \mathrm{C}$. Subsequently, the membranes were incubated for $2 \mathrm{~h}$ with a horseradish peroxidase-conjugated immunoglobulin G secondary antibody (cat. no. ab205718; 1:5,000 dilution; Abcam) at room temperature. Enhanced chemiluminescence western blotting detection reagent (GE Healthcare Life Sciences, Little Chalfont, UK) was applied to the membranes for visualization of the protein signals. The densitometry of protein signals was analyzed with Quantity One software version 4.62 (Bio-Rad Laboratories, Inc.).

Statistical analysis. All statistical analyses were performed using the SPSS 18.0 statistics software (SPSS, Inc.). All data are presented as mean \pm standard deviation of three independent replicates. Correlations between the OSER1-AS1 level and clinicopathological factors in patients with NSCLC were examined using the $\chi^{2}$ test. Differences among $\geq 3$ groups were evaluated using one-way analysis of variance followed by Tukey's test. Comparisons of data between two groups were performed using Student's t-test. Correlations between the expression levels of OSER1-AS1 and miR-433-3p in the NSCLC tissues were assessed using Spearman's correlation analysis. Overall survival curves were plotted using the Kaplan-Meier method and compared using the log-rank test. A P-value of $<0.05$ was considered to indicate statistical significance.

\section{Results}

OSER1-AS1 is induced in NSCLC tissues and cell lines. To determine the OSER1-AS1 expression status in NSCLC, its expression was evaluated in 53 pairs of NSCLC tissues and adjacent normal tissues using RT-PCR. OSER1-AS1 expression was significantly upregulated in the NSCLC tissues compared with that in the adjacent normal tissues (Fig. 1A). OSER1-AS1 expression was also evaluated in various NSCLC cell lines using RT-qPCR, with BEAS-2B cells used as the control. Notably, OSER1-AS1 expression was significantly higher in all five NSCLC cell lines than in the BEAS-2B cells (Fig. 1B). In summary, OSER1-AS1 expression is upregulated in both NSCLC tissues and cell lines.

Clinical value of OSERI-ASI in patients with NSCLC. Based on the median OSER1-AS1 expression value in NSCLC tissues, all 53 NSCLC tissues collected from the enrolled patients were classified into either a low $(\mathrm{n}=26)$ or high $(\mathrm{n}=27)$ OSER1-AS1 expression group. $\chi^{2}$ test revealed obvious correlations between OSER1-AS1 expression and tumor size $(\mathrm{P}=0.024)$, TNM stage $(\mathrm{P}=0.028)$, and lymph node metastasis $(\mathrm{P}=0.045)$ in the 53 NSCLC tissues (Table I). Additionally, Kaplan-Meier analysis verified that the high-OSER1-AS1 expression group had a shorter overall survival than the low-OSER1-AS1 expression group (Fig. 1C, P=0.0253). The abovementioned data imply that OSER1-AS1 plays important roles during NSCLC tumorigenesis and progression.

Silencing of OSER1-AS1 restrains NSCLC cell proliferation, migration, and invasion and promotes cell apoptosis. $\mathrm{H} 522$ and A549 cells exhibited the highest OSER1-AS1 expression among the five NSCLC cell lines; therefore, they were selected for 
A

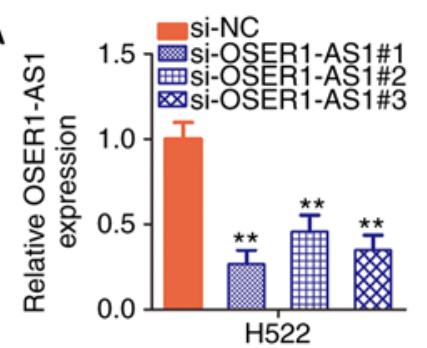

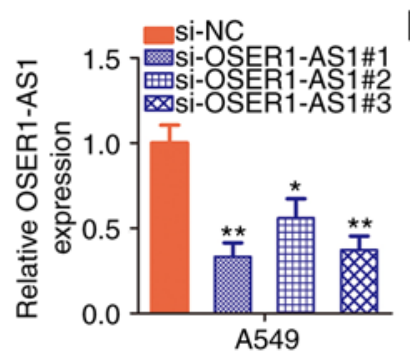
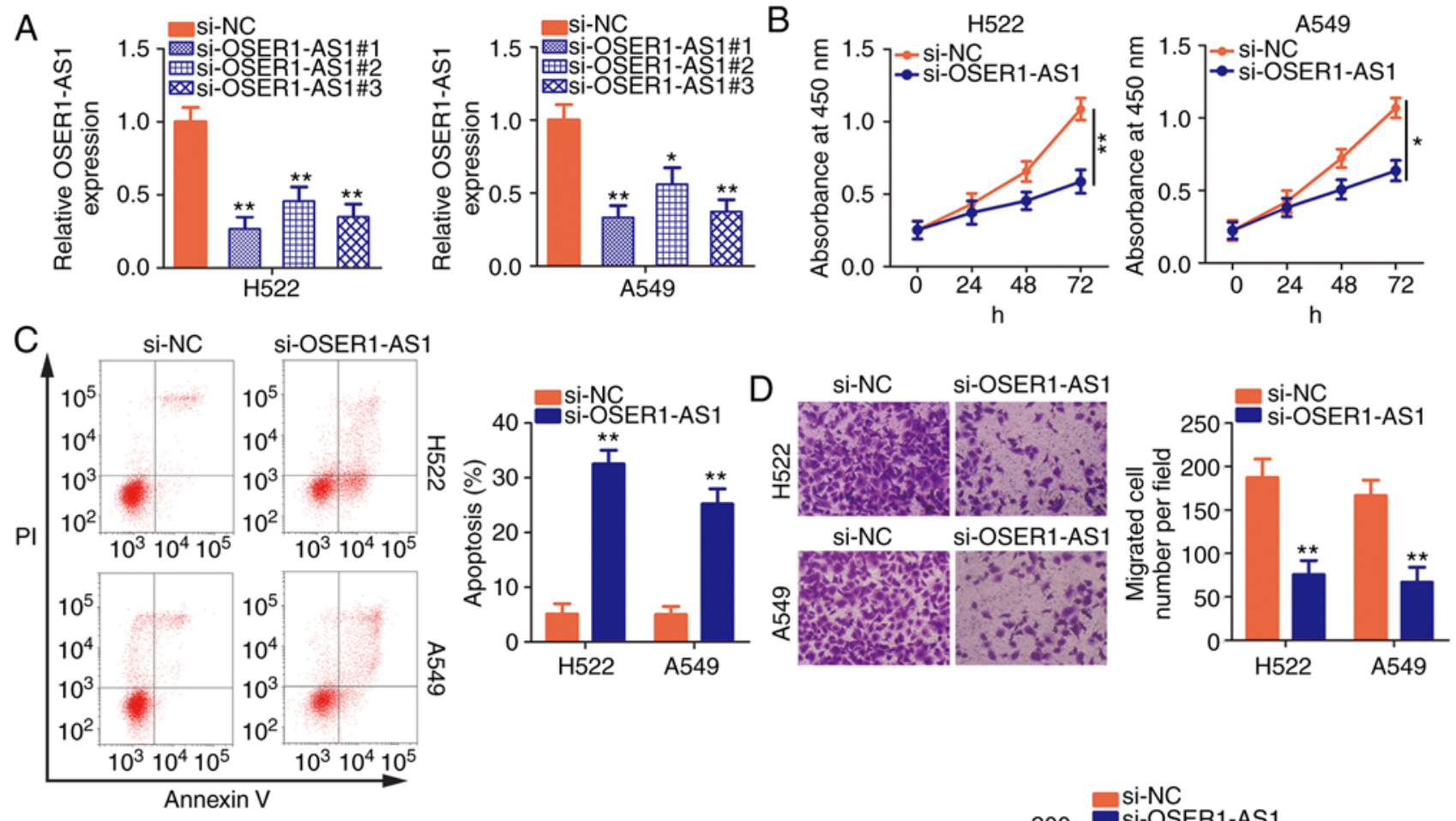

E

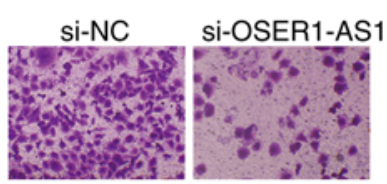

$\mathrm{H} 522$

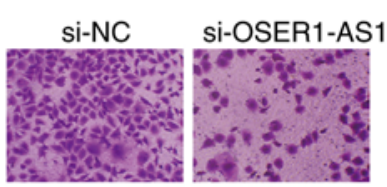

A549

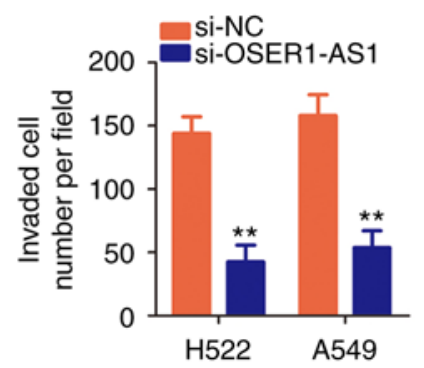

Figure 2. Inhibition of the long noncoding RNA OSER1-AS1 suppresses the malignant biological phenotypes of NSCLC. (A) Determination of OSER1-AS1 expression in H522 and A549 cell lines after si-NC or si-OSER1-AS1 transfection using RT-qPCR. (B and C) Cell Counting Kit-8 assay and flow cytometry for the determination of cell proliferation and apoptosis, respectively, in H522 and A549 cells after OSER1-AS1 knockdown. (D and E) Cell migration and invasion assays of $\mathrm{H} 522$ and A549 cells transfected with small interfering (si)-OSER1-AS1 or control si-NC. ${ }^{*} \mathrm{P}<0.05$ and ${ }^{* *} \mathrm{P}<0.01$, compared to the si-NC group. OSER1-AS1, RNA OSER1 antisense RNA 1; NSCLC, non-small cell lung cancer; RT-qPCR, reverse transcription-quantitative polymerase chain reaction; PI, propidium iodide.

further functional studies of the roles of OSER1-AS1 in NSCLC. Three siRNAs were designed to disturb OSER1-AS1 expression in H522 and A549 cells, and interference efficiency was assessed using RT-qPCR. Notably, si-OSER1-AS1\#1 (renamed as si-OSER-AS1) most efficiently silenced OSER1-AS1 expression in H522 and A549 cells (Fig. 2A) and was chosen for endogenous knockdown experiments. Using the CCK-8 assay, we demonstrated that OSER1-AS1 knockdown significantly decreased the proliferative abilities of H522 and A549 cells (Fig. 2B). In addition, si-OSER1-AS1 transfection significantly increased the apoptosis rate in both cell lines (Fig. 2C), as evidenced by flow cytometry. Furthermore, the migratory and invasion abilities of these cells were assessed by cell migration and invasion assays, respectively. The numbers of migrated (Fig. 2D) and invaded (Fig. 2E) H522 and A549 cells were significantly reduced after si-OSER1-AS1 transfection, thus confirming that OSER1-AS1 silencing suppressed the migratory and invasion abilities of these cells. Taken together, these data indicate that OSER1-AS1 exerts cancer-promoting actions in NSCLC progression.

OSER1-AS1 functions as a molecular sponge for miR-433-3p in NSCLC cells. The specific roles of lncRNAs are determined by their localization (29). To illustrate the mechanism by which OSER1-AS1 regulates the biological behaviors of NSCLC, we first analyzed the distribution of expression of this lncRNA in NSCLC cells. Nuclear and cytoplasmic RNA fractionation indicated that OSER1-AS1 was mostly localized in the cytoplasm of H522 and A549 cells (Fig. 3A), suggesting that this lncRNA functions as a ceRNA or molecular sponge for certain miRNAs.

According to Starbase3.0, OSER1-AS1 harbors base pairs complementary to miR-433-3p (Fig. 3B), and the latter miRNA was chosen for subsequent experimental identification because of its role as a cancer inhibitor during NSCLC progression (30). Luciferase reporter and RIP assays were performed to validate the true interaction between OSER1-AS1 and miR-433-3p in NSCLC cells. First, the efficiency of the miR-433-3p mimic was confirmed through RT-qPCR, and the results indicated that transfection with the mimic significantly increased miR-433-3p expression in H522 and A549 cells (Fig. 3C). Luciferase reporter assay revealed an evident decrease in the luciferase activity of OSER1-AS1-wt in H522 and A549 cells by upregulation of miR-433-3p expression compared with that in the miR-NC group, whereas no significant change 

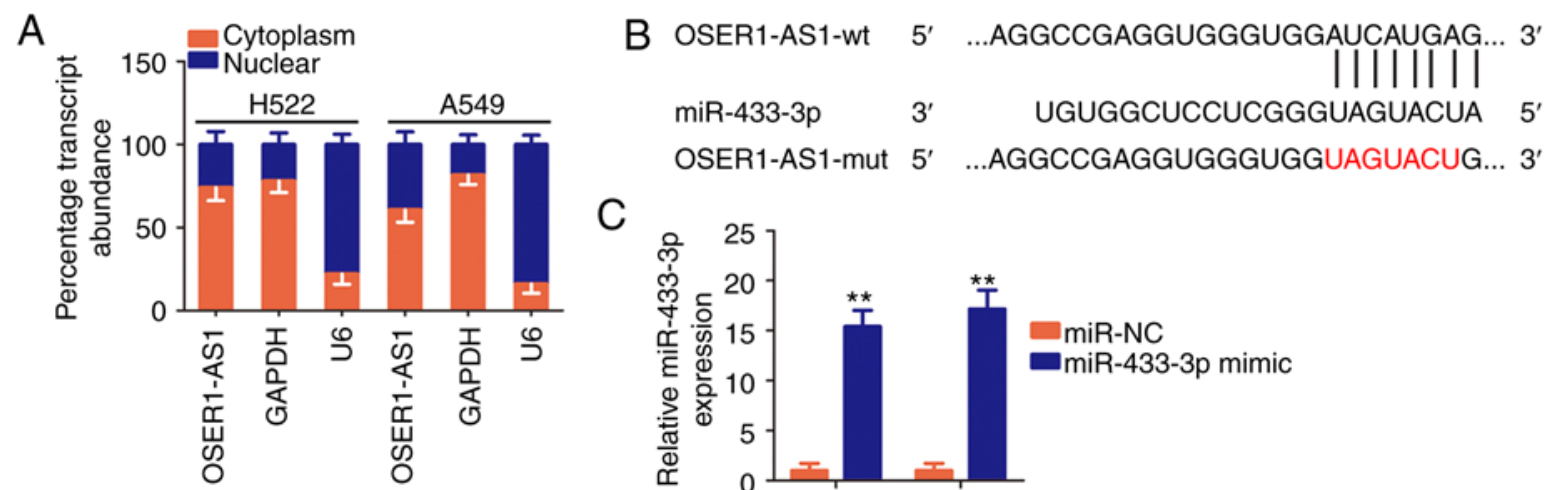

C
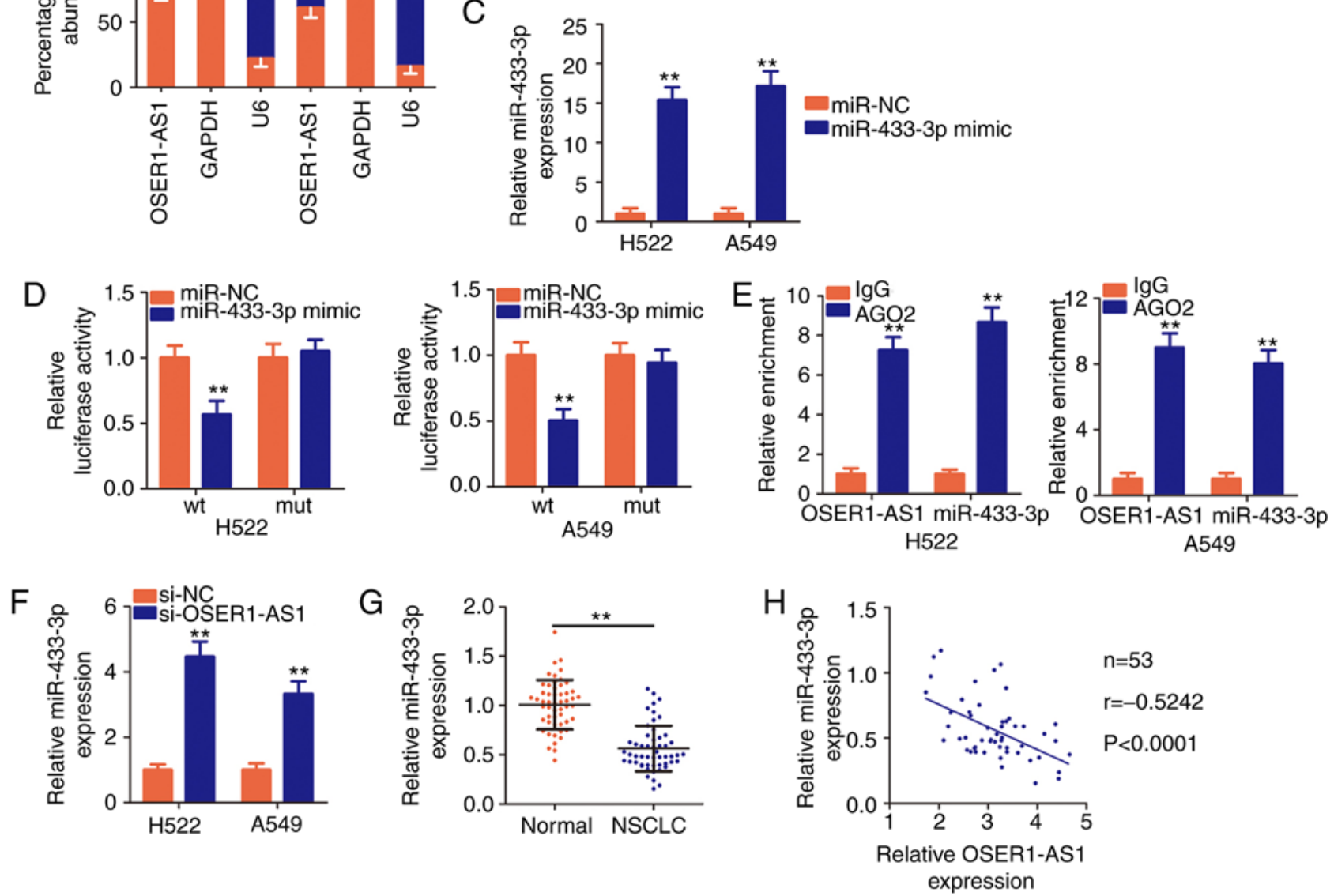

Figure 3. The long noncoding RNA OSER1-AS1 acts as a molecular sponge for miR-433-3p in NSCLC cells. (A) Nuclear and cytoplasmic RNA fractionation to determine the localization of OSER1-AS1 in H522 and A549 cells. (B) A graphical representation of wild-type (wt) and mutant (mut) miR-433-3p-binding sites on OSER1-AS1. (C) Determination of miR-433-3p expression in H522 and A549 cells transfected with miR-433-3p mimic or miR-NC. ${ }^{* *} \mathrm{P}<0.01$, compared with the miR-NC group. (D) Detection of the binding interaction between miR-433-3p and OSER1-AS1 in H522 and A549 cells by luciferase reporter assay. H522 and A549 cells were cotransfected with miR-433-3p mimic or miR-NC and OSER1-AS1-wt or OSER1-AS1-mut plasmids. Transfected cells were harvested after $48 \mathrm{~h}$ and subjected to luciferase activity assay. ${ }^{* *} \mathrm{P}<0.01$, compared with the miR-NC group. (E) Evaluation of the enrichment of miR-433-3p and OSER1-AS1 expression in H522 and A549 cells by RNA immunoprecipitation assay with Ago2 or IgG antibodies and subsequent RT-qPCR. ${ }^{* *} \mathrm{P}<0.01$, compared with the IgG group. (F) Quantification of miR-433-3p in OSER1-AS1-depleted H522 and A549 cells using RT-qPCR. "P<0.01, compared with the si-NC group. (G) Detection of miR-433-3p expression in 53 pairs of NSCLC tissues and adjacent normal tissues using RT-qPCR. ${ }^{* *} \mathrm{P}<0.01$, compared with adjacent normal tissues. (H) Spearman's correlation analysis of the correlation between OSER1-AS1 and miR-433-3p expression in 53 NSCLC tissues ( $r=-0.5242$, $\mathrm{P}<0.0001)$. OSER1-AS1, RNA OSER1 antisense RNA 1; miR, microRNA; NSCLC, non-small cell lung cancer; RT-qPCR, reverse transcription-quantitative polymerase chain reaction.

was observed in OSER1-AS1-mut reporter plasmid activity after miR-433-3p overexpression (Fig. 3D). In addition, RIP assay demonstrated notable abundance of OSER1-AS1 and miR-433-3p in Ago2-containing beads compared with that in the $\mathrm{IgG}$ controls, suggesting that miR-433-3p can directly bind to OSER1-AS1 in NSCLC cells (Fig. 3E).

The effects of OSER1-AS1 expression on miR-433-3p expression in NSCLC cells were evaluated using RT-qPCR. miR-433-3p expression was significantly increased in the H522 and A549 cells upon OSER1-AS1 knockdown (Fig. 3F). Furthermore, RT-qPCR data revealed that miR-433-3p was weakly expressed in the NSCLC tissues compared with that noted in the adjacent normal tissues (Fig. 3G). Concordantly, Spearman's correlation analysis revealed an inverse correlation between the expression of OSER1-AS1 and miR-433-3p in the
$53 \mathrm{NSCLC}$ tissues (Fig. 3H; $\mathrm{r}=-0.5242$, $\mathrm{P}<0.0001$ ). In general, these results suggest that OSER1-AS1 acts as a ceRNA and molecular sponge for miR-433-3p in NSCLC cells.

OSER1-AS1 positively controls Smad2 expression in NSCLC cells by sponging miR-433-3p. Given that OSER1-AS1 functions as a ceRNA in NSCLC, we hypothesized that it modulates the expression of mRNAs targeted by miR-433-3p. Because a previous study identified $S m a d 2$ as a direct target of miR-433-3p in NSCLC cells (30), we next evaluated Smad2 mRNA and protein expression in OSER1-AS1-depleted H522 and A549 cells. RT-qPCR and western blotting revealed that silencing of OSER1-AS1 expression decreased Smad2 expression in the H522 and A549 cells at both the mRNA (Fig. 4A) and protein (Fig. 4B) levels. To further assess the relationship between 
A

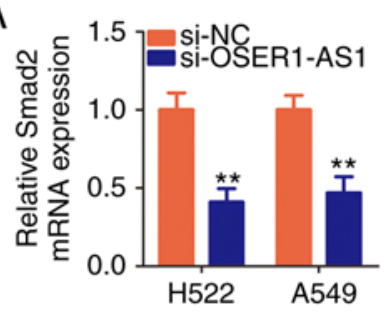

D

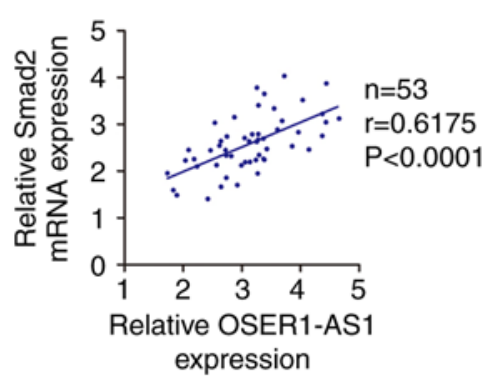

G

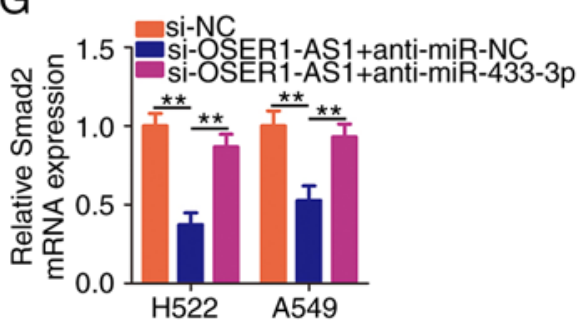

B

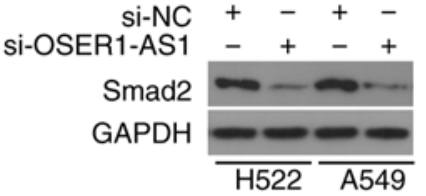

E

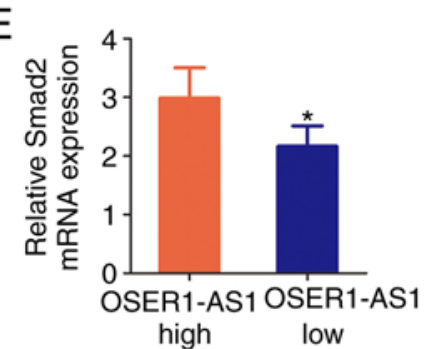

$\mathrm{H}$

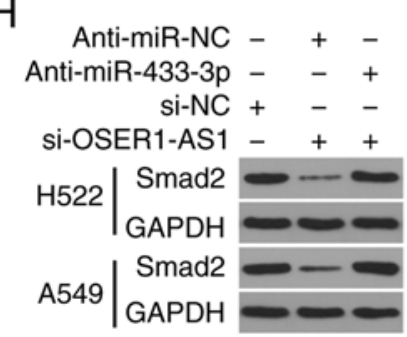

C
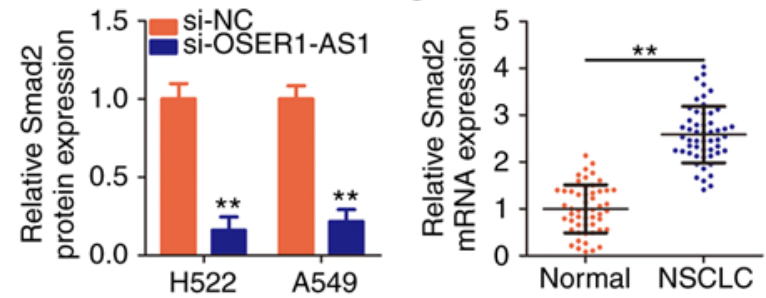
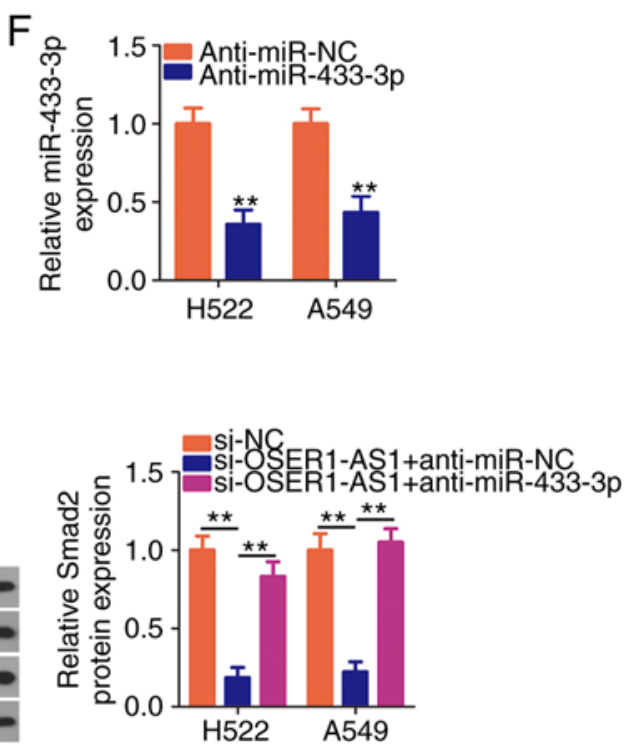

Figure 4. OSER1-AS1 knockdown suppresses Smad2 expression in NSCLC cells. (A and B) Detection of Smad2 mRNA and protein expression in OSER1-AS1-deficient H522 and A549 cell lines by RT-qPCR and western blotting, respectively. ${ }^{* *} \mathrm{P}<0.01$, compared with the si-NC group. (C) Determination of Smad2 mRNA expression in 53 pairs of NSCLC tissues and adjacent normal tissues using RT-qPCR. ${ }^{* *} \mathrm{P}<0.01$. (D) Spearman's correlation analysis of the correlation between OSER1-AS1 and Smad2 mRNA expression in the 53 NSCLC tissues ( $\mathrm{r}=0.6175, \mathrm{P}<0.0001)$. (E) Comparison of Smad2 expression in NSCLC tissues derived from patients in the high- and low-OSER1-AS1 expression groups. $\mathrm{P}<0.05$, compared with the OSER1-AS1 high group. (F) miR-433-3p expression in $\mathrm{H} 522$ and A549 cells transfected with anti-miR-433-3p or anti-miR-NC using RT-qPCR. ${ }^{* *} \mathrm{P}<0.01$, compared with the anti-miR-NC group. $(\mathrm{G}$ and $\mathrm{H}$ ) Cotransfection of OSER1-AS1-deficient H522 and A549 cells with anti-miR-433-3p or anti-miR-NC and detection of Smad2 protein expression using RT-qPCR and western blotting, respectively. ${ }^{* *} \mathrm{P}<0.01$. OSER1-AS1, RNA OSER1 antisense RNA 1; Smad2, mothers against decapentaplegic homolog 2; miR, microRNA; NSCLC, non-small cell lung cancer; RT-qPCR, reverse transcription-quantitative polymerase chain reaction.

OSER1-AS1 and miR-433-3p expression in NSCLC cells, we performed RT-qPCR to measure $S m a d 2$ expression in 53 pairs of NSCLC tissues and adjacent normal tissues. Smad2 expression was significantly higher in the NSCLC tissues than that noted in the adjacent normal tissues (Fig. 4C). Furthermore, Smad2 mRNA expression was positively correlated with OSER1-AS1 expression in the 53 NSCLC tissues (Fig. 4D; r=0.6175, $\mathrm{P}<0.0001)$. Furthermore, higher Smad2 mRNA expression was observed in the high-OSER1-AS1 expression group than in the low-OSER1-AS1 expression group (Fig. 4E).

Rescue assays were performed to determine whether OSER1-AS1 acts as a regulator of Smad2 expression in NSCLC cells by sponging miR-433-3p. We first determined the transfection efficiency of a miR-433-3p inhibitor (anti-miR-433-3p) using RT-qPCR (Fig. 4F). Subsequently, anti-miR-433-3p or anti-miR-NC was introduced together with si-OSER1-AS1 into H522 and A549 cells, after which RT-qPCR and western blotting were performed to determine any changes in Smad2 mRNA and protein expressions, respectively. Notably, OSER1-AS1 knockdown led to a considerable decrease in Smad2 mRNA (Fig. 4G) and protein (Fig. 4H) expression, whereas the inhibitory impact of the knockdown was reversed by miR-433-3p inhibition. In general, OSER1-AS1 acts as a ceRNA for miR-433-3p and thereby increases Smad2 expression in NSCLC cells.

miR-433-3p inhibition or Smad2 restoration counteracts the suppressive effects of OSERI-AS1 knockdown on NSCLC cell proliferation, migration, and invasion and promoted cell apoptosis. We next performed rescue assays to confirm whether OSER1-AS1 affected the malignant properties of NSCLC cells by regulating the miR-433-3p/Smad 2 axis. Here, we introduced si-OSER1-AS1 with anti-miR-433-3p or anti-miR-NC into H522 and A549 cells. CCK-8 assay revealed that cotransfection with anti-miR-433-3p neutralized the inhibitory effect of OSER1-AS1 interference on H522 and A549 cell proliferation (Fig. 5A). Additionally, OSER1-AS1-deficient H522 and A549 cells exhibited increased apoptosis, which was reversed after miR-433-3p inhibition as demonstrated by flow cytometry (Fig. 5B). Furthermore, the reintroduction of anti-miR-433-3p abolished the effects of OSER1-AS1 knockdown on the migration (Fig. 5C) and invasiveness (Fig. 5D) of H522 and A549 cells.

Transfection with pc-Smad2 led to a noticeable increase in Smad2 mRNA (Fig. 6A) and protein (Fig. 6B) expression in the H522 and A549 cells, as revealed using RT-qPCR and 

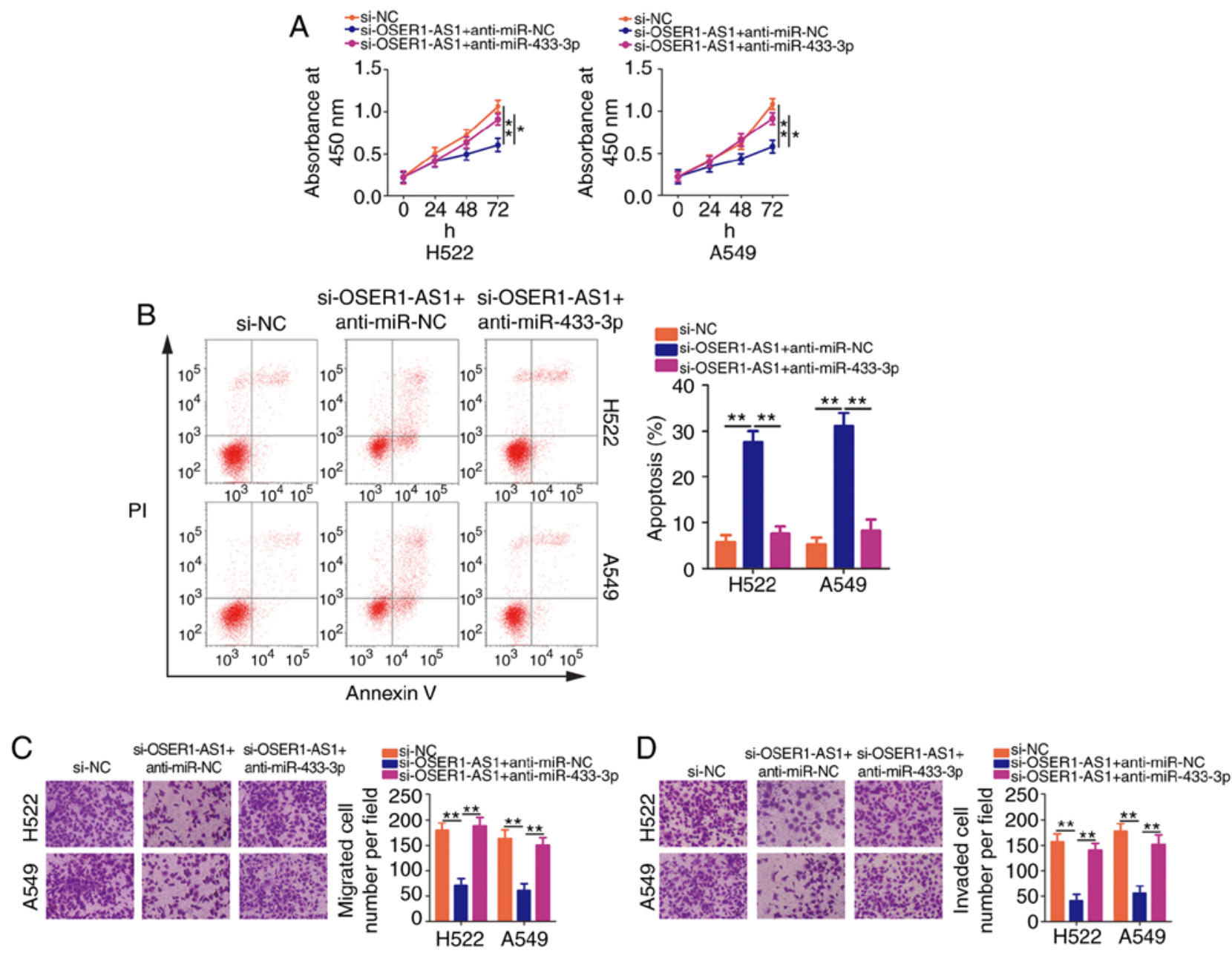

Figure 5. miR-433-3p knockdown abrogates the effects of OSER1-AS1 depletion on the malignant phenotypes of NSCLC cells. H522 and A549 cell lines were cotransfected with si-OSER1-AS1 and anti-miR-433-3p or anti-miR-NC. (A) Cell proliferation, (B) apoptosis, (C) migration, and (D) invasion of the cotransfected cells were determined using Cell Counting Kit-8 assay, flow cytometry, and cell migration assay, and cell invasion assay, respectively. "P<0.05 and ${ }^{* *} \mathrm{P}<0.01$. miR, microRNA; OSER1-AS1, RNA OSER1 antisense RNA 1; NSCLC, non-small cell lung cancer; PI, propidium iodide.

western blotting, respectively. Additionally, rescue assays were performed on H522 and A549 cells after cotransfection with si-OSER1-AS1 and pc-Smad2 or pcDNA3.1. Similarly, the restoration of Smad2 expression in H522 and A549 cells reversed the suppressed proliferation (Fig. 6C), promoted apoptosis (Fig. 6D) as well as impaired migration (Fig. 6E) and invasion (Fig. 6F) induced by OSER1-AS1 knockdown. In summary, OSER1-AS1 exerts its pro-oncogenic roles in NSCLC cells by regulating the miR-433-3p/Smad 2 axis.

OSERI-AS1 knockdown restrains the growth of NSCLC cells in vivo. The effects of OSER1-AS1 inhibition on NSCLC growth in vivo were further investigated by tumor xenograft assay. The interference efficiency of sh-OSER1-AS1 in H522 cells was verified using RT-qPCR (Fig. 7A). Subsequently, H522 cells that stably expressed sh-OSER1-AS1 or sh-NC were inoculated subcutaneously into nude mice. The tumor xenografts in the sh-OSER1-AS1 group were smaller in size and volume than those in the sh-NC group (Fig. 7B and C). Additionally, the weight of tumor xenografts was significantly lower in the sh-OSER1-AS1 group than that in the sh-NC group (Fig. 7D). All mice were euthanized after 5 weeks, and the tumor xenografts were dissected and analyzed to determine
OSER1-AS1, miR-433-3p, and Smad2 expression. RT-qPCR revealed the continued suppression of OSER1-AS1 (Fig. 7E) and upregulation of miR-433-3p (Fig. 7F) expression in the tumor xenografts of mice injected with H522 cells that stably expressed OSER 1-AS1. Furthermore, Smad2 protein expression was significantly reduced in the tumor xenografts that originated from H522 cells stably transfected with sh-OSER1-AS1 (Fig. 7G). These results suggest that OSER1-AS1 knockdown impedes the growth of NSCLC cells in vivo by upregulating miR-433-3p and downregulating $\operatorname{Smad} 2$ expression.

\section{Discussion}

Many recent studies have demonstrated the abnormal expression of various long noncoding RNAs (lncRNAs) in non-small cell lung cancer (NSCLC) (31-33). These dysregulated lncRNAs act as important regulators of the pathogenesis of NSCLC and have crucial effects on NSCLC tumorigenesis, progression, and metastasis (34-36). Therefore, a comprehensive study of the functional involvement of lncRNAs in NSCLC may yield useful information and help identify attractive targets for anticancer management. To date, more than 3,000 IncRNAs have been validated in the human genome, but only 

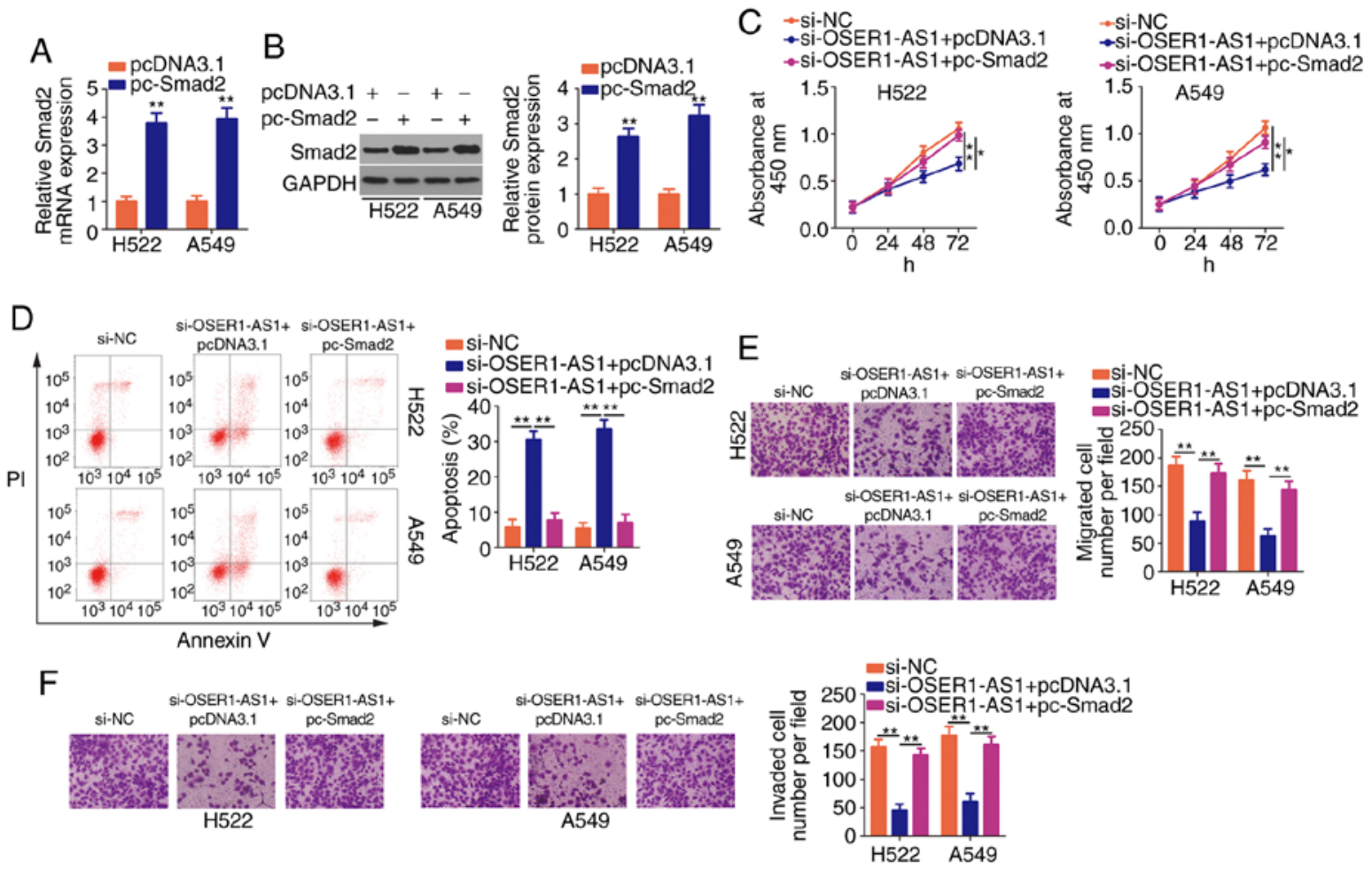

Figure 6. Reintroduction of Smad2 reverses the effects of OSER1-AS1 depletion on NSCLC cells. (A and B) Determination of pc-Smad2 transfection efficiency in H522 and A549 cells by RT-qPCR and western blotting. ${ }^{* *} \mathrm{P}<0.01$, compared with the pcDNA3.1 group. (C and D) Cotransfection of H522 and A549 cells with si-OSER1-AS1 and pc-Smad2 or empty pcDNA3.1 and subsequent evaluation of cell proliferation and apoptosis by Cell Counting Kit-8 assay and flow cytometry, respectively. ${ }^{*} \mathrm{P}<0.05$ and ${ }^{* *} \mathrm{P}<0.01$. (E and F) Evaluation of cell migration and invasion in the aforementioned cells by cell migration and invasion assays, respectively. ${ }^{* *} \mathrm{P}<0.01$. OSER1-AS1, RNA OSER1 antisense RNA 1; Smad2, mothers against decapentaplegic homolog 2; NSCLC, non-small cell lung cancer; RT-qPCR, reverse transcription-quantitative polymerase chain reaction; PI, propidium iodide.

A

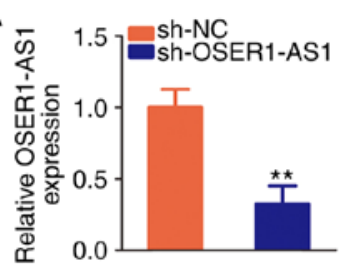

$\mathrm{E}$

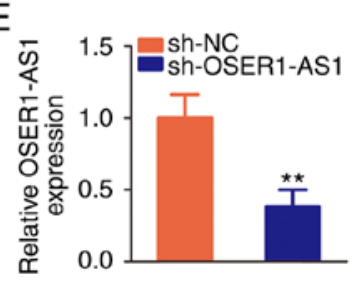

B

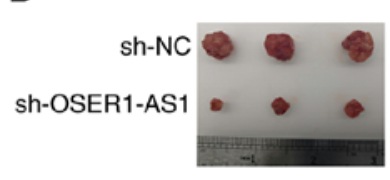

$\mathrm{F}$

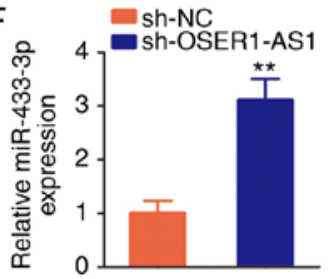

C
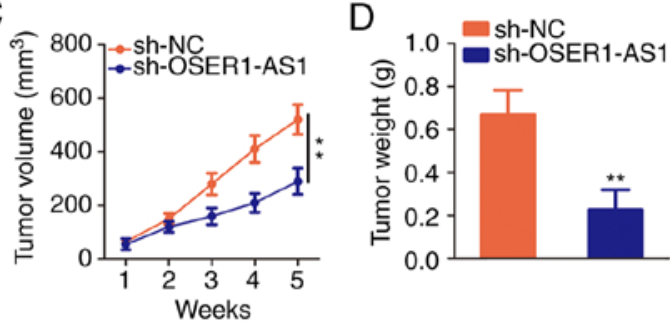

G

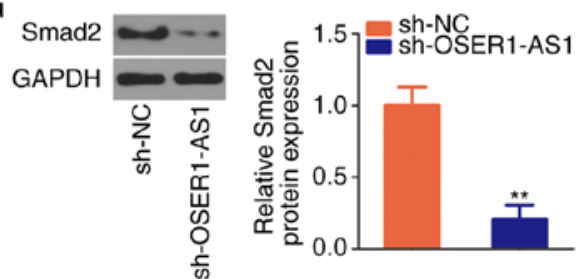

Figure 7. Depletion of OSER1-AS1 suppresses the growth of NSCLC xenograft tumors in vivo. (A) Detection of OSER1-AS1 expression in sh-OSER1-AS1 or sh-NC-stably transected H522 cells by RT-qPCR. ${ }^{* *} \mathrm{P}<0.01$, compared with the sh-NC group. (B) Images of tumor xenografts derived from sh-OSER1-AS1 or sh-NC-stably transected H522 cells. (C) Plots of tumor xenograft growth according to tumor volume. ${ }^{* *} \mathrm{P}<0.01$. (D) The weights of tumor xenografts from the sh-OSER1-AS1 and sh-NC groups. ${ }^{* *} \mathrm{P}<0.01$, compared with the sh-NC group. (E and F) Detection of OSER1-AS1 and miR-433-3p expression in tumor xenografts by RT-qPCR. ${ }^{* *} \mathrm{P}<0.01$, compared with the sh-NC group. (G) Isolation of total protein from tumor xenografts in the sh-OSER1-AS1 and sh-NC groups and measurement of Smad2 protein expression by western blotting. ${ }^{* *} \mathrm{P}<0.01$, compared with the sh-NC group. OSER1-AS1, RNA OSER1 antisense RNA 1; Smad2, mothers against decapentaplegic homolog 2; NSCLC, non-small cell lung cancer; RT-qPCR, reverse transcription-quantitative polymerase chain reaction; miR, microRNA.

few of them have been studied (37). In this study, we evaluated OSER1 antisense RNA 1 (OSER1-AS1) expression in NSCLC tissues and cell lines and explored the clinical value of aberrantly high OSER1-AS1 expression in NSCLC. Furthermore, we conducted a series of functional experiments to explore the detailed roles of OSER1-AS1 in NSCLC progression. Moreover, we thoroughly elucidated the downstream target of OSER1-AS1 and its underlying mechanisms. 
The oncogenic action of OSER1-AS1 in hepatocellular carcinoma has been well studied. In this tumor type, OSER1-AS1 expression is upregulated and strongly associated with adverse clinicopathological factors and low disease-free survival and overall survival (24). Functionally, interference with OSER1-AS1 expression in hepatocellular carcinoma cells suppressed their proliferation, migration, and invasion and promoted their apoptosis (24). To the best of our knowledge, no previous study has investigated the expression patterns and detailed functions of OSER1-AS1 in NSCLC. Herein, we collected 53 pairs of NSCLC tissues and adjacent normal tissues as well as determined OSER1-AS1 expression using RT-qPCR. Our results revealed strong expression of OSER1-AS1 in the NSCLC tissues. Consistently, all five tested NSCLC cell lines exhibited relatively higher OSER1-AS1 expressions than human nontumorigenic bronchial epithelial BEAS-2B cells. High OSER1-AS1 expression presented an obvious correlation with tumor size, TNM stage, lymph node metastasis and shorter overall survival in patients with NSCLC. Functional research affirmed that OSER1-AS1 knockdown restricted NSCLC cell proliferation, migration, and invasion in vitro; promoted cell apoptosis; and restrained tumor growth in vivo.

The mechanistic events by which IncRNAs participate in carcinogenesis and cancer progression depend on their subcellular localization in tumor cells (29). Accordingly, we first determined the expression distribution of OSER1-AS1 in NSCLC cells through nuclear and cytoplasmic RNA fractionation. OSER1-AS1 was found to be mostly located in the cytoplasm of NSCLC cells. Increasing evidence suggests that cytoplasmic lncRNAs can act as competitive endogenous RNAs (ceRNAs) and thereby competitively bind to the target sites of miRNAs, leading to the release of target mRNAs $(38,39)$.

In this study, we used the StarBase 3.0 database to identify potential miRNAs as targets for sponging by OSER1-AS1. Bioinformatics analysis indicated that OSER1-AS1 contained base pairs complementary to miR-433-3p. Next, we conducted a series of experiments to validate our hypothesis. Luciferase reporter and RIP assays further verified that OSER1-AS1 bound directly to miR-433-3p in NSCLC cells. Furthermore, miR-433-3p expression was drastically downregulated in NSCLC tissues, and this expression pattern exhibited an inverse correlation with that of OSER1-AS1. Moreover, OSER1-AS1 knockdown led to increased miR-433-3p expression and decreased Smad2 (a direct target gene of miR-433-3p) expression in NSCLC cells, whereas miR-433-3p inhibition counteracted the regulatory effect of OSER1-AS1 knockdown on Smad2 expression. Therefore, the oncogenic role of OSER1-AS1 in NSCLC can be explained in the context of a ceRNA pathway involving OSER1-AS1, miR-433-3p and Smad2.

Fan et al reported that OSER1-AS1 performs oncogenic roles in hepatocellular carcinoma by regulating the miR-372-3p/Rab23 axis (24). miR-372-3p is upregulated in NSCLC and exerts tumor-suppressive actions on cell growth and metastasis (40). Hence, the miR-372-3p/Rab23 axis could not be the downstream regulatory mechanism of OSER1-AS1 in NSCLC. In this study, our new findings revealed different working mechanisms, which were not consistent with previous studies regarding OSER1-AS1 (24).
Aberrant expression of miR-433-3p has been observed in multiple human cancer types including NSCLC (30). In our study, miR-433-3p overexpression led to obvious decreases in NSCLC cell proliferation, migration, and invasion in vitro and hindered tumor growth in vivo. Mechanistically, Smad2 has been verified as a direct downstream target of miR-433-3p in NSCLC cells. Smad2, a member of the Smad family, is a key signal transducer and transcriptional modulator implicated in the regulation of multiple signaling pathways (41). Smad2 expression is upregulated in NSCLC, where it modulates various cancer-associated aggressive processes (42-44). These observations highlight the importance of the miR-433-3p/Smad2 axis in NSCLC tumorigenesis and progression. Nevertheless, the upstream mechanism that regulates this axis remains largely unclear and warrants further investigation. Our results demonstrated an apparent increase in Smad2 expression in NSCLC tissues as well as its positive correlation with OSER1-AS1 expression. Our restoration experiments revealed that enhanced activity of the miR-433-3p/Smad2 axis alleviated the inhibitory effects of OSER1-AS1 knockdown on NSCLC cell proliferation, migration, and invasion and reversed its apoptosis-enhancing effect. These results collectively imply that the tumor-promoting actions of OSER1-AS1 in NSCLC cells may be attributable to the miR-433-3p-induced upregulation of Smad2 expression.

In the present research, we did not analyze the driver oncogene mutations in the NSCLC tissues; hence, statistical analysis could not be performed to test the comparisons between OSER1-AS1 expression and oncogene mutations in NSCLC. This is a limitation of our study, and we will resolve the limitation in the near future.

OSER1-AS1 drives the malignant properties of NSCLC cells in vitro and in vivo by regulating the miR-433-3p/Smad 2 axis. Identification of the OSER1-AS1/miR-433-3p/Smad 2 pathway offers a novel perspective on the discovery of effective therapeutic and diagnostic targets for NSCLC.

\section{Acknowledgements}

Not applicable.

\section{Funding}

No funding was received.

\section{Availability of data and materials}

The datasets used and/or analyzed during the present study are available from the corresponding author on reasonable request.

\section{Authors' contributions}

QZ and XL conceived and designed the study. XL, SH and YG carried out the experiments. QZ and XL wrote the paper. All authors reviewed and edited the manuscript. All authors read and approved the manuscript and agree to be accountable for all aspects of the research in ensuring that the accuracy or integrity of any part of the work are appropriately investigated and resolved. 


\section{Ethics approval and consent to participate}

The present study was approved by the Research Ethics Committee of Weifang People's Hospital (Weifang, China), and was performed in accordance with the Declaration of Helsinki and the guidelines of the Ethics Committee of The Seventh People's Hospital. Written informed consent was obtained from all patients for the use of their clinical tissues.

\section{Patient consent for publication}

Not applicable.

\section{Competing interests}

The authors declare that they have no competing interests.

\section{References}

1. Siegel RL, Miller KD and Jemal A: Cancer statistics, 2019. CA Cancer J Clin 69: 7-34, 2019.

2. Bray F, Ferlay J, Soerjomataram I, Siegel RL, Torre LA and Jemal A: Global cancer statistics 2018: GLOBOCAN estimates of incidence and mortality worldwide for 36 cancers in 185 countries. CA Cancer J Clin 68: 394-424, 2018.

3. Shin JY, Yoon JK and Marwaha G: Progress in the treatment and outcomes for Early-Stage non-small cell lung cancer. Lung 196: 351-358, 2018

4. Lagos GG, Izar B and Rizvi NA: Beyond tumor PD-L1: Emerging genomic biomarkers for checkpoint inhibitor immunotherapy. Am Soc Clin Oncol Educ Book 40: 1-11, 2020.

5. Travis WD: Lung cancer pathology: Current concepts. Clin Chest Med 41: 67-85, 2020.

6. Vansteenkiste J, Crino L, Dooms C, Douillard JY, Faivre-Finn C, Lim E, Rocco G, Senan S, Van Schil P, Veronesi G, et al: 2nd ESMO Consensus Conference on lung cancer: Early-stage non-small-cell lung cancer consensus on diagnosis, treatment and follow-up. Ann Oncol 25: 1462-1474, 2014.

7. Pirker R: Adjuvant chemotherapy in patients with completely resected non-small cell lung cancer. Transl Lung Cancer Res 3 : 305-310, 2014

8. Torre LA, Siegel RL and Jemal A: Lung cancer statistics. Adv Exp Med Biol 893: 1-19, 2016.

9. Brambilla E and Gazdar A: Pathogenesis of lung cancer signalling pathways: Roadmap for therapies. Eur Respir J 33: 1485-1497, 2009

10. Kadara H, Scheet P, Wistuba II and Spira AE: Early events in the molecular pathogenesis of lung cancer. Cancer Prev Res (Phila) 9: 518-527, 2016

11. Peng WX, Koirala P and Mo YY: LncRNA-mediated regulation of cell signaling in cancer. Oncogene 36: 5661-5667, 2017.

12. Ponting CP, Oliver PL and Reik W: Evolution and functions of long noncoding RNAs. Cell 136: 629-641, 2009.

13. Huarte M: The emerging role of lncRNAs in cancer. Nat Med 21: $1253-1261,2015$

14. Cheetham SW, Gruhl F, Mattick JS and Dinger ME: Long noncoding RNAs and the genetics of cancer. Br J Cancer 108 2419-2425, 2013.

15. Ma HP, Wang LX, Li W, Guo HH, Wu Y and Li XY: Upregulation of LINC00504 is associated with aggressive progression and poor prognosis in non-small cell lung cancer. Eur Rev Med Pharmacol Sci 24: 699-703, 2020.

16. Qiu C, Li S, Sun D and Yang S: lncRNA PVT1 accelerates progression of non-small cell lung cancer via targeting miRNA-526b/EZH2 regulatory loop. Oncol Lett 19: 1267-1272, 2020.

17. Wang Z, Zhang J, Yang B, Li R, Jin L, Wang Z, Yu H, Liu C, Mao Y and You Q: Long Intergenic noncoding RNA 00261 acts as a tumor suppressor in non-small cell lung cancer via regulating miR-105/FHL1 Axis. J Cancer 10: 6414-6421, 2019.

18. Shangguan WJ, Liu HT, Que ZJ, Qian FF, Liu LS and Tian JH: TOB1-AS1 suppresses non-small cell lung cancer cell migration and invasion through a ceRNA network. Exp Ther Med 18: 4249-4258, 2019.
19. Gao YP, Li Y, Li HJ and Zhao B: LncRNA NBR2 inhibits EMT progression by regulating Notch1 pathway in NSCLC. Eur Rev Med Pharmacol Sci 23: 7950-7958, 2019.

20. Wang Y, Luo X, Liu Y, Han G and Sun D: Long noncoding RNA RMRP promotes proliferation and invasion via targeting miR-1-3p in non-small-cell lung cancer. J Cell Biochem 120: 15170-15181, 2019.

21. Bian C, Yuan L and Gai H: A long non-coding RNA LINC01288 facilitates non-small cell lung cancer progression through stabilizing IL-6 mRNA. Biochem Biophys Res Commun 514: 443-449, 2019.

22. Calin GA and Croce CM: MicroRNA signatures in human cancers. Nat Rev Cancer 6: 857-866, 2006.

23. Cesana M, Cacchiarelli D, Legnini I, Santini T, Sthandier O, Chinappi M, Tramontano A and Bozzoni I: A long noncoding RNA controls muscle differentiation by functioning as a competing endogenous RNA. Cell 147: 358-369, 2011.

24. Fan J, Zhang J, Huang S and Li P: lncRNA OSER1-AS1 acts as a ceRNA to promote tumorigenesis in hepatocellular carcinoma by regulating miR-372-3p/Rab23 axis. Biochem Biophys Res Commun 521: 196-203, 2020.

25. Sibio S, Sica GS, Di Carlo S, Cardi M, Di Giorgio A, Sollazzo BM and Sammartino P: Surgical treatment of intraperitoneal metastases from lung cancer: Two case reports and a review of the literature. J Med Case Rep 13: 262, 2019.

26. Yi E, Kim D, Cho S, Kim K and Jheon S: Clinical outcomes of cytoreductive surgery combined with intrapleural perfusion of hyperthermic chemotherapy in advanced lung adenocarcinoma with pleural dissemination. J Thorac Dis 8: 1550-1560, 2016.

27. Livak KJ and Schmittgen TD: Analysis of relative gene expression data using real-time quantitative PCR and the 2(-Delta Delta C(T)) method. Methods 25: 402-408, 2001.

28. Li JH, Liu S, Zhou H, Qu LH and Yang JH: starBase v2.0: Decoding miRNA-ceRNA, miRNA-ncRNA and protein-RNA interaction networks from large-scale CLIP-Seq data. Nucleic Acids Res 42 (Database Issue): D92-D97, 2014

29. Wang L, Cho KB, Li Y, Tao G, Xie Z and Guo B: Long Noncoding RNA (lncRNA)-mediated competing endogenous RNA networks provide novel potential biomarkers and therapeutic targets for colorectal cancer. Int J Mol Sci 20: 5758, 2019.

30. Li J, Chen M and Yu B: miR-433 suppresses tumor progression via Smad2 in non-small cell lung cancer. Pathol Res Pract 215: 152591, 2019.

31. Tang L, Wang T, Zhang Y, Zhang J, Zhao H, Wang H, Wu Y and Liu K: Long non-coding RNA AWPPH promotes postoperative distant recurrence in resected non-small cell lung cancer by upregulating transforming growth factor beta 1 (TGF- $\beta 1$ ). Med Sci Monit 25: 2535-2541, 2019.

32. Bai Y, Zhang G, Chu H, Li P and Li J: The positive feedback loop of lncRNA DANCR/miR-138/Sox4 facilitates malignancy in non-small cell lung cancer. Am J Cancer Res 9: 270-284, 2019.

33. Liu X, Lu X, Zhen F, Jin S, Yu T, Zhu Q, Wang W, Xu K, Yao J and Guo R: LINC00665 induces acquired resistance to Gefitinib through recruiting EZH2 and activating PI3K/AKT pathway in NSCLC. Mol Ther Nucleic Acids 16: 155-161, 2019.

34. Zhang Y, Li Y, Han L, Zhang P and Sun S: SUMO1P3 is associated clinical progression and facilitates cell migration and invasion through regulating miR-136 in non-small cell lung cancer. Biomed Pharmacother 113: 108686, 2019.

35. Zhang B, Wang H, Wang Q, Xu J, Jiang P and Li W: Knockout of lncRNA UCA1 inhibits drug resistance to gefitinib via targeting STAT3 signaling in NSCLC. Minerva Med 110: 273-275, 2019.

36. Li B, Gu W and Zhu X: NEAT1 mediates paclitaxel-resistance of non-small cell of lung cancer through activation of Akt/mTOR signalling pathway. J Drug Target 27: 1061-1067, 2019.

37. Hutchinson JN, Ensminger AW, Clemson CM, Lynch CR, Lawrence JB and Chess A: A screen for nuclear transcripts identifies two linked noncoding RNAs associated with SC35 splicing domains. BMC Genomics 8: 39, 2007.

38. Wang JJ, Huang YQ, Song W, Li YF, Wang H, Wang WJ and Huang M: Comprehensive analysis of the $\operatorname{lncRN}$-associated competing endogenous RNA network in breast cancer. Oncol Rep 42: 2572-2582, 2019.

39. Xu F, Zhao Y, Qin G, Huan Y, Li L and Gao W: Comprehensive analysis of competing endogenous RNA networks associated with cholangiocarcinoma. Exp Ther Med 18: 4103-4112, 2019.

40. Wang Q, Liu S, Zhao X, Wang Y, Tian D and Jiang W: MiR-372-3p promotes cell growth and metastasis by targeting FGF9 in lung squamous cell carcinoma. Cancer Med 6: 1323-1330, 2017. 
41. Kamato D and Little PJ: Smad2 linker region phosphorylation is an autonomous cell signalling pathway: Implications for multiple disease pathologies. Biomed Pharmacother 124: 109854,2020

42. Chen T,Zhu J, Cai T, Du W, Zhang Y,Zhu Q, Liu Z and Huang JA: Suppression of non-small cell lung cancer migration and invasion by hsa-miR-486-5p via the TGF- $\beta /$ SMAD2 signaling pathway. J Cancer 10: 6014-6024, 2019.

43. Othman $\mathrm{N}$ and Nagoor NH: Overexpression of miR3615p plays an oncogenic role in human lung adenocarcinoma through the regulation of SMAD2. Int J Oncol 54: 306-314, 2019.
44. Wang Z, Lu Y, Sheng B, Ding Y and Cheng X: Catalpol inhibits TGF- $\beta 1$-induced epithelial-mesenchymal transition in human non-small-cell lung cancer cells through the inactivation of Smad2/3 and NF- $\mathrm{B}$ B signaling pathways. J Cell Biochem: Sep 11, 2018 doi: 10.1002/jcb.27535 (Epub Ahead of Print).

(i) (3) This work is licensed under a Creative Commons Attribution-NonCommercial-NoDerivatives 4.0 International (CC BY-NC-ND 4.0) License. 